\title{
A Rank-Dependent Expected Utility Model for Strategic Route Choice with Stated Preference Data
}

\author{
Michael Razo* Song $\mathrm{Gao}^{\dagger}$
}

September 7, 2011

\begin{abstract}
Route choice behavior under real-time traffic information needs to be adequately modeled for the proper analysis of a transportation system in the presence of Advanced Traveler Information Systems (ATIS). This paper focuses on strategic route choice, where a traveler is able to plan ahead for traffic information that $\mathrm{s} / \mathrm{he}$ will receive in the future. A stated preference (SP) survey was conducted with interactive maps showing two types of networks with risky travel times, one type eliciting risk attitude and the other allowing for strategic route choice with a detour to an incident-prone road segment and real-time traffic information. The preliminary analysis suggests that a traveler's risk attitude is probability-dependent. A rank-dependent expected utility (RDEU) model is adopted to account for such a phenomenon, where the decision weight of a probabilistic outcome depends on its ranking among all outcomes and a non-linear transformation of the cumulative probability. A latent-class mixed Logit model for panel data is specified with a RDEU component and two latent classes, strategic and non-strategic route choice. The estimated strategic class probability is significantly different from 0 and 1 respectively, suggesting that a route choice model under real-time information should consider both types of behavior. The estimated RDEU parameters show significant diminishing sensitivities to both outcome and probability and explain the probability-dependent risk attitude.
\end{abstract}

Keywords Strategic route choice; Rank-dependent expected utility; Cumulative prospect theory; Advanced Traveler Information System; Latent-class model; Discrete choice model; Mixed Logit; Panel data.

\footnotetext{
${ }^{*}$ Research Assistant, University of Massachusetts, Amherst, MA 01003. E-mail: mrazo@ecs.umass.edu

${ }^{\dagger}$ Assistant Professor, University of Massachusetts, Marston Hall 214C, 130 Natural Resources Rd, Amherst, MA 01003, USA. Tel: +1 413-545-2688. E-mail: songgao@ecs.umass.edu
} 


\section{Introduction}

With advances in surveillance, telecommunication and personal electronic device technologies, real-time traffic information has increasingly become available to travelers through, for example, smartphones equipped with location services, or in-vehicle GPS units subscribing to real-time traffic information. Such information is valuable because it could potentially help reduce uncertainties in the transportation system due to accidents, fluctuating demand, vehicle breakdown, road construction, bad weather, and other incidents. It follows that effective analysis and prediction of traffic demand in the presence of Advanced Traveler Information Systems (ATIS) requires proper accounting for the effects of real-time information on individual route choice behavior.

En route information on revealed traffic conditions could potentially prompt travelers to change route. For example, a variable message sign (VMS) indicating an incident downstream along the freeway could induce some travelers to take an earlier exit and follow a parallel arterial. This type of on-the-spot response to real-time information has been studied extensively in the literature (for recent reviews, see Abdel-Aty and Abdalla, 2006; Chorus et al., 2006). Travelers are assumed to make successive path choices at intermediate decision nodes, where the attributes of the alternative paths might be updated due to the revealed realtime traffic conditions. The availability of the information and any detours does not affect the traveler's route choice until the information is actually revealed.

However, travelers need not only respond to information on the spot; they can also plan ahead for information that will be available in the future. Suppose a traveler is faced with a choice of two branches of roads, one with no real-time information, and the other presenting information to travelers at certain points in the network where detours are available. Even though the real-time information itself is not available to the traveler when s/he must choose a branch, the knowledge that such information will be available can affect the traveler's choice.

Strategic thinking, in this context, refers to the traveler's recognition that certain detours can be taken in response to future information. The perceived quality of a route can be greatly affected by this consideration, and subjects whose choices reflect this are considered to exhibit strategic choice behavior.

Discrete choice models for strategic route choice are developed in Gao et al. (2008) and Gao et al. (2010) and their differences from conventional non-strategic route choice and/or expected utility models compared. Later on empirical evidence of strategic route choice behavior is presented in Razo and Gao (2010), where a simple hypothetical network is implemented as an interactive graphical map. Subjects use the map to make route choices based on travel times and real-time information. Subjects' choices are analyzed using risk and travel time metrics, and 
a latent-class Logit model is estimated with the mean travel time and travel time standard deviation as explanatory variables. The data analysis and model estimation both supported the hypothesis that some subjects did in fact account for future information when choosing a route.

Another interesting finding from Razo and Gao (2010) is that travelers' risk attitudes changed with the probability of travel delay. The developed Logit systematic utility function thus includes three different parameters for the travel time standard deviation, one for each of the three levels of delay probabilities used in the survey. The model fits the data satisfactorily, however it cannot be used for prediction where the delay probability can be any value.

In this paper, we adopt a rank-dependent expected utility (RDEU) specification of the systematic utility function to model travelers' subjective perception of probabilities. RDEU is proposed by Quiggin (1982) and one of the most popular descriptive models of decision under risk. The better known cumulative prospect theory (CPT) by Tversky and Kahneman (1992) is a combination of their original prospect theory (Kahneman and Tversky, 1979) and RDEU. In RDEU, the decision weight of an outcome is determined not only by its own probability, but also the probabilities of outcomes that rank higher. A commonly accepted inverted Sshaped weighting function (Tversky and Kahneman, 1992) is used in this paper together with a power function for the value function. A similar model is applied to analyze airline route choice in de Lapparent (2010).

The key difference between the RDEU framework and the CPT framework is that RDEU does not compare potential outcomes to a reference point. In the RDEU models developed here, all outcomes are considered losses. We do not adopt the reference-dependent CPT in this paper, mainly because it is not clear whether any reference points other than 0 exist in our route choice survey, or how they could be determined if so. de Palma et al. (2008) suggest that the determination of reference points is one of the major obstacles to the application of CPT and that they likely vary over individuals as well as choice contexts. Most previous CPTbased empirical travel studies either present the survey questions explicitly in terms of gains or losses (see, e.g. Katsikopoulos et al., 2000; Katsikopoulos et al., 2002; Schwanen and Ettema, 2009; Xu et al., 2010), possess natural reference points (the preferred arrival time in departure time choice) (see, e.g. Senbil and Kitamura, 2004; Jou et al., 2008), or assume a reference point arbitrarily (see, e.g. Avineri, 2004; Avineri and Prashker, 2005; Ben-Elia and Shiftan, 2010). Alternatively the model developed in this paper can be viewed as a loss-only CPT model with 0 as the reference point. Similarly, Gao et al. (2010) presents a loss-only CPT model validated through synthetic data, yet using the lowest origin-destination travel time as the reference point.

The remainder of the paper is organized as follows. Section 2 describes the 
survey set up and preliminary findings with regards to strategic route choice and risk attitudes. The RDEU model applied to travel time outcomes is presented next in Section 3. A latent-class mixed Logit model with panel data is specified in Section 4, with two latent classes: strategic and non-strategic decisions. Section 5 presents the estimation results of the parameters for the value and weighting functions as well as the latent strategic class probability. Conclusions and future directions are given in Section 6.

\section{Stated Preference (SP) Survey}

\subsection{Survey Design}

The survey was designed to measure two aspects of the subjects' route choice behavior: risk attitude and strategic thinking. Since strategic route choice is only relevant in networks with risky travel times, it is crucial to properly understand and model risk behavior when addressing strategic thinking.

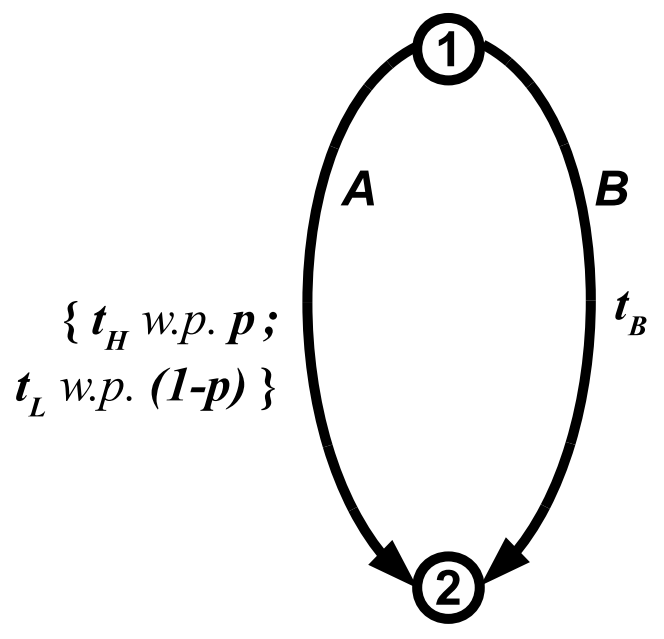

Figure 1: Abstract network for simple risk map type

To this end, the survey was designed using two map types: a "simple risk" map and a "strategy" map. The simple risk map (Figure 1) presents subjects with a clear choice between a path with deterministic travel time (Link B, the safe route) and a path with random travel time (Link A, the risky route). Since there are no detours or real-time information available, there is no opportunity for strategic choice, and this map type measures a subject's risk attitude only. A wide range of travel time scenarios is presented using the same map topology, to collect a large number of 
data points for each subject.

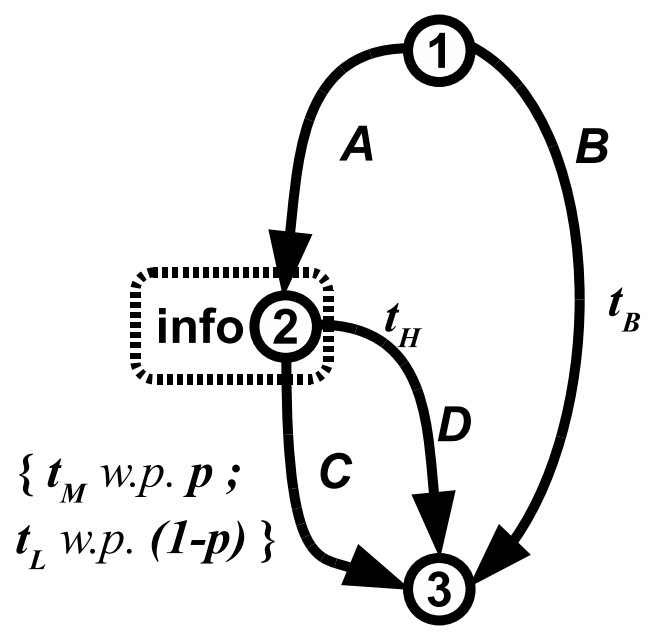

Figure 2: Abstract network for routing strategy tests

The strategy map extends the simple risk map by adding a detour (Link D) and real-time information (Figure 2, "info" at Node 2) to the risky alternative. The realtime information notifies the subject of the actual travel time on Link $\mathrm{C}$, before s/he must decide whether link to take out of Node 2. This allows the subject to choose the faster of Links $C$ and $D$ and avoid large delays. The map can therefore measure the extent to which a subject recognizes and utilizes strategically advantageous real-time information.

There are two critical design elements of the strategy map. First, the strategy map is designed such that the user's first movement is effectively a choice between a safe route and a risky branch. Since strategic choice is distinguished by consideration of information not yet received, any choices made after the information is received are irrelevant. In this case, the first movement is the only relevant choice.

Second, each scenario presented in the strategy map is designed to correspond with a scenario presented in the simple risk map. Travel times are designed such that a strategic subject will perceive exactly the same risk on the risky branch of the strategy map as s/he did on the risky route of the corresponding simple map.

Specifically, $t_{L}$ is the lower potential travel time of the risky link, while $t_{H}$ is the deterministic travel time on the detour. $t_{M}$, the higher potential travel time of the risky link, is always exceedingly larger than any other travel time on the map. This means that a subject who heeds the real-time information will never take the risky link when the higher travel time is realized.

A perfectly strategic subject would recognize this advantage even before the 
information is received and perceive the outlook of the risky branch of the strategy map as identical to that of the risky path in the corresponding simple map.

A non-strategic subject, however, will perceive a much higher potential delay $\left(t_{M}\right)$ in the strategy map, which would be reflected in a decreased willingness to choose the risky alternative in the strategy map than in the simple map.

\subsection{Interface}

The survey was conducted using interactive graphical maps with a point-and-click interface (example screenshot in Figure 3). The survey was presented to subjects as a graphical map. This approach was chosen because of the simplicity and clarity as compared to describing the scenarios in written or verbal form. The visual format provides an easily comprehensible representation of each scenario, and helps the user relate to the intended context.

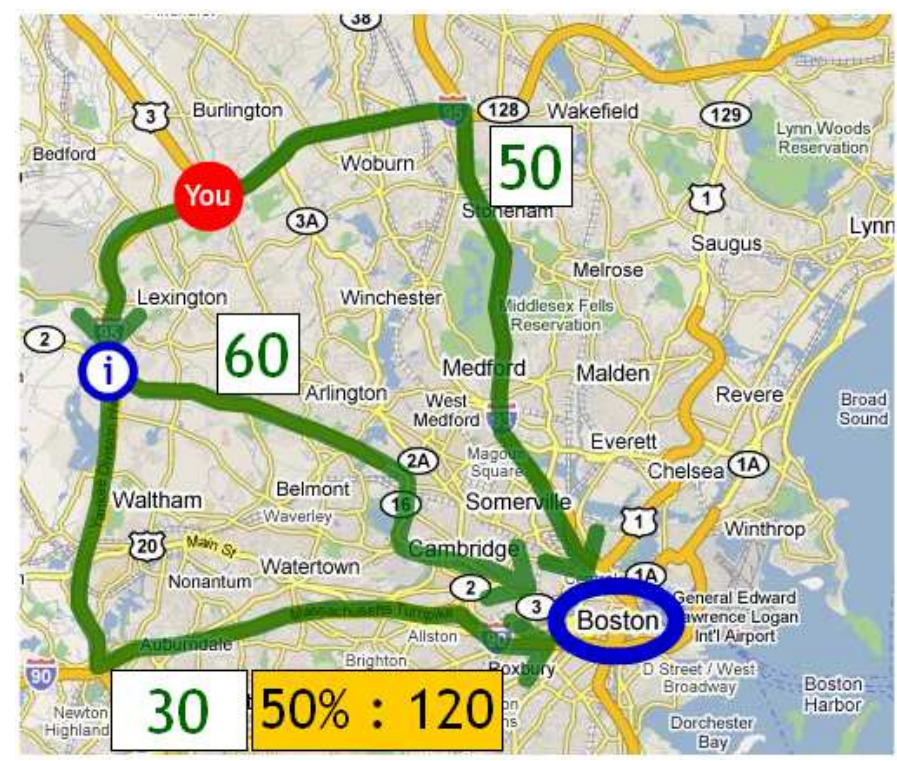

Figure 3: Example map interface with information and detour

The white labels in Figure 3 indicate the usual travel time of the adjacent route. The risky link has an additional yellow label to indicate the probability of a delay and the full travel time of the route in the event of a delay. The travel time between the origin and the information node is negligible. In maps without real-time information, the actual travel time will only be revealed if and when the user travels across the link. 
For maps that include real-time information, a blue "i" icon is shown at the node where the user will receive the information. When the user arrives at that node, the actual travel time of the risky link is revealed, and the user may choose whether to use the risky link or the detour.

Survey sessions were guided by a coordinator, with thorough verbal and written instructions. After completing several warm-up scenarios, each with an introduction from the coordinator, subjects completed six groups of scenarios, with breaks between each. Each group corresponded to a combination of map type and probability of the delay $(20 \%, 50 \%$ or $80 \%)$. Within each group, travel times were generated by a factorial design, with $t_{H}$ and $t_{B}$ as the design factors. $t_{L}$ was fixed at 30 minutes throughout all scenarios. $t_{H}$ took the values 40,50 and $60 ; t_{B}$ took values from 35 to $t_{H}-5$, such that the safe route was not dominated by the risky branch. In the strategy map scenarios, the high time on the risky link, $t_{M}$, was fixed at 120 minutes. The coordinator introduced each scenario group to provide context and highlight any changes. Sessions typically lasted 40 to 60 minutes, including all preparation and paperwork.

\subsection{Participants}

The survey was taken by 74 individual subjects, with a total of over 3400 observations. Subjects were recruited from the University of Massachusetts student and staff community, as well as the surrounding area. The mean age was 24.2 years and mean driving experience was 6.9 years. $54 \%$ of the subjects were male, $46 \%$ female. In an exit questionnaire taken after all maps were completed, subjects answered two yes-or-no questions probing whether they had chosen routes strategically in the survey, along with a free-response question for explanation:

1. When you left the start point (your first movement), did the possibility of a 120-minute travel time on I-90 convince you to use I-93 instead?

2. Did the availability of the information sign affect this decision?

3. Why or why not? (referring to Question 2)

Based on the two yes-or-no answers, each user's response was categorized as clearly strategic (Q1:No, Q2:Yes), clearly non-strategic (Q1:Yes, Q2:No), or indeterminate (Q1,Q2:Yes or Q1,Q2:No). 37 responses (50\%) were considered clearly strategic, while 10 responses $(13.5 \%)$ were considered clearly non-strategic. 27 responses $(36.5 \%)$ were indeterminate. The free responses to Question 3 were simply reviewed to verify that these classifications generally matched the subjects' apparent thinking process. The questionnaire results were not used in modeling or 
any other quantitative analysis, but provide an informal sanity check against the analytical results.

\subsection{Risk Attitude Findings}

Analysis of each individual's choice behavior was performed by defining benefitto-risk ratio for the risky alternative in each scenario. Benefit is defined as travel time of the safe alternative minus the travel time of the risky alternative. This can be considered the expected savings in travel time gained by taking the risky alternative. Risk is defined as the standard deviation of the travel time on the risky alternative.

A positive benefit/risk ratio means that the expected travel time of the risky alternative is lower (better) than that of the safe alternative. A negative ratio indicates that expected travel time of the risky alternative is higher (worse). Generally speaking, a higher ratio suggests a more "worthwhile" risky alternative. By definition, a risk-averse subject will only choose the risky alternative if its expected travel time is better than that of the safe alternative, and thus the benefit/risk ratio is positive. A risk-seeking subject, however, might choose the risky alternative even when its expected travel time is worse than that of the safe alternative. The sign of the benefit/risk ratio therefore indicates whether a choice could have resulted from risk-averse behavior.

Each subject displayed a minimum threshold, below which s/he never chose the risky alternative, and a maximum threshold, above which s/he always chose the risky alternative. Between these two thresholds is an "ambivalent range" in which the subject's choice behavior was not always consistent with respect to the benefit/risk ratio. Figures 4, 5, and 6 show the ambivalent ranges of each subject, for each level of delay probability. Subjects with ambivalent ranges entirely below zero displayed completely risk-prone behavior, while those with ranges entirely above zero showed completely risk-averse behavior. Those with ambivalent ranges extending both above and below zero displayed both risk-seeking behavior in some scenarios and risk-averse behavior in others.

The most striking finding from analysis of the ambivalent ranges is that the probability of delay significantly impacts choice behavior, with subjects becoming more risk-seeking at higher probabilities and more risk-averse at lower probabilities. At $p=0.2,88 \%$ of subjects displayed entirely risk-averse behavior, meaning they never chose a risky alternative with a negative benefit/risk ratio. At $p=0.5$, $86 \%$ percent of subjects displayed entirely risk-neutral or risk-averse behavior. At $p=0.8,80 \%$ of subjects displayed entirely risk-seeking behavior, meaning they consistently accept the risk even though the benefit-risk ratio is negative. This effect was also confirmed with statistical significance using a mean-standard devi- 


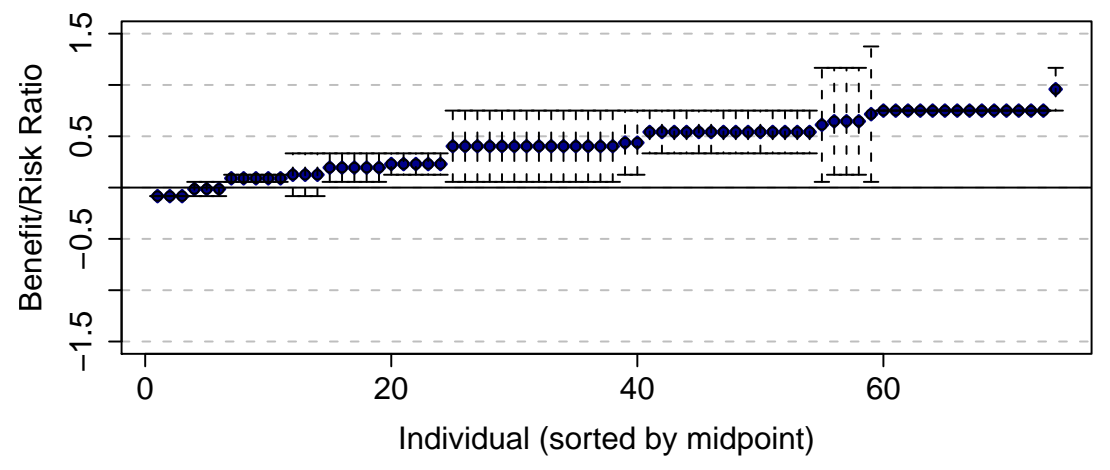

Figure 4: Ambivalent range for each user, for scenarios with $p=0.2$

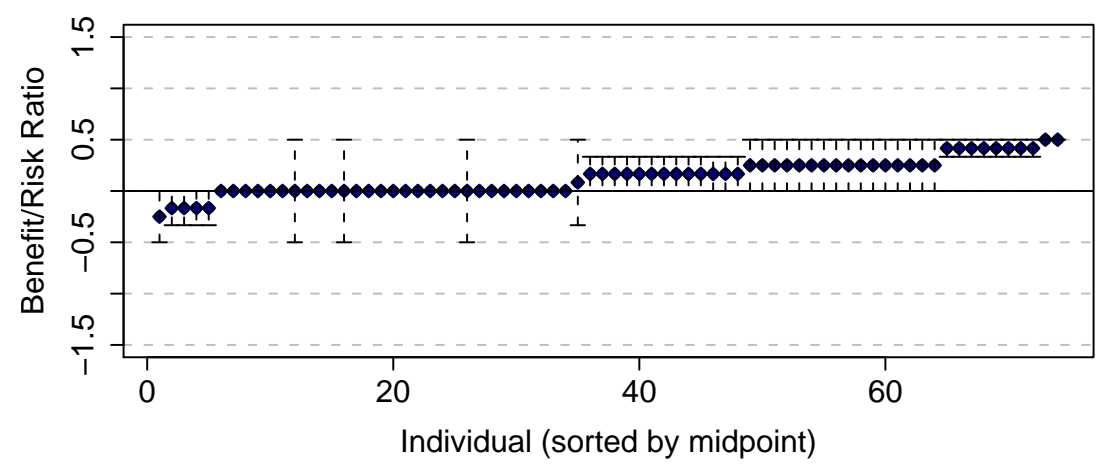

Figure 5: Ambivalent range for each user, for scenarios with $p=0.5$

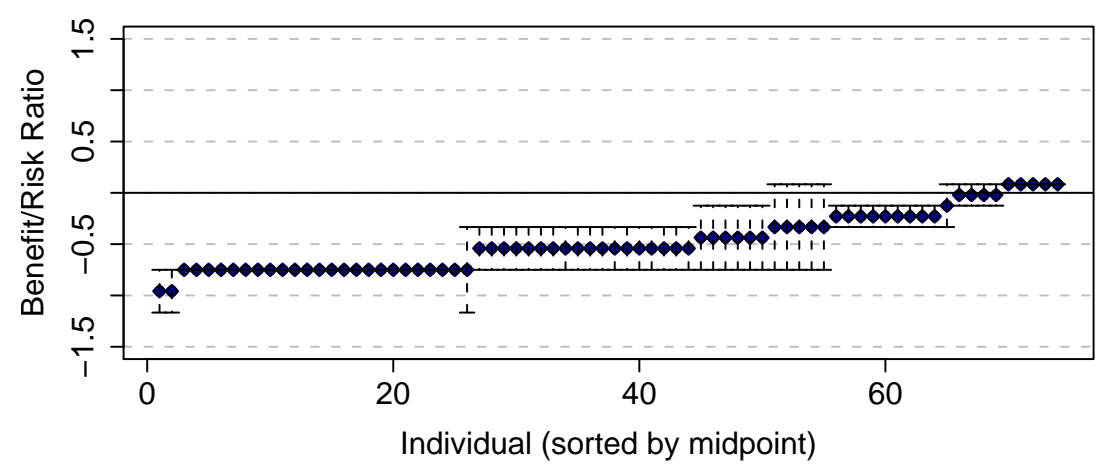

Figure 6: Ambivalent range for eaçh user, for scenarios with $p=0.8$ 
ation model with dummy variables to measure risk attitude at the three levels of delay probability presented in the survey (Razo and Gao, 2010). The estimated risk parameter was negative (showing risk aversion) for probability 0.2 and not significantly different from 0 for probability 0.5 , but becomes large and positive (risk-seeking) for probability 0.8 .

This observation is consistent with the findings of Avineri and Prashker (2004). The effect is likely the result of a mismatch between subjects' intuition and the true probability of delay. Specifically, subjects may be overestimating the likelihood of a delay when $p=0.2$ but underestimating when $p=0.8$. The resulting behavior is a general increase in risk-seeking behavior as the probability of delay increases.

\subsection{Strategic Choice Findings}

As described above, each strategy map was designed to correspond directly to a simple risk map, so that a subject's choice behavior can be compared between the two map types. Specifically, the strategy map is designed so that a perfectly strategic subject would perceive exactly the same prospects in the strategy map as in the simple risk map. A non-strategic subject, however, would fail to recognize the irrelevance of the large potential delay of the strategy map, and would therefore be less likely to accept the risk.

It is therefore expected that highly strategic subjects will frequently choose the risky alternative in strategy maps where they have also done so for the corresponding simple map. Less strategic subjects, by contrast, will frequently choose the risky alternative in the simple map yet reject it in the corresponding strategy map.

A straightforward comparison of a single subject's choices between the two map types provides an informative look at the subject's level of strategic behavior. Consistency of choice between the two map types, when the risky alternative is chosen, can be used as a simple measure of strategic tendency. $78.43 \%$ of subjects displayed a consistency of 0.5 or better. $37.25 \%$ displayed 0.75 or better consistency. Just three subjects - or 5.88\% - displayed perfect consistency (1.0). 11.76\% of subjects displayed very poor consistency, less than 0.25 . Since the distribution is clearly skewed toward greater-than- 0.5 consistency, this analysis suggests that strategic behavior is prevalent in our data.

In assessing the observations irrespective of the subject, it was found that $31 \%$ of all observations are distinguishably strategic while $15 \%$ are distinguishably nonstrategic. The remainder are not distinguishable as the user did not select the risky alternative in the simple map. 


\section{Rank-Dependent Expected Utility (RDEU) Model}

Considering the strong evidence that probability of delay has an impact on risk attitude, our next natural step is to pursue a model capable of accounting for this behavior. The model developed in this paper is based on the Rank-Dependent Expected Utility (RDEU) theory (Quiggin, 1982; Schmeidler, 1989), which is one of the most popular non-expected utility (non-EU) theories among economists (Starmer, 2000).

Consider a prospect $\mathbf{q}$ of risky travel times (outcomes) with their corresponding objective probabilities $\mathbf{q}=\left\{x_{1}, p_{1} ; x_{2}, p_{2} ; \ldots ; x_{m}, p_{m}\right\}$, where travel times $x_{i}$ are represented by negative numbers and sorted by extremity, such that $x_{1}$ is the lowest travel time (largest negative number) and $x_{m}$ the highest (smallest negative number). Travel times are treated as negative numbers to be consistent with theories in economics, where payoffs are generally used as outcomes and treated as positive numbers. A theory for decision under risk assigns a value to a prospect through a preference function $V(\mathbf{q})$ and assumes a decision maker chooses the prospect with the largest $V($.$) .$

The conventional expected utility theory (EUT) transforms the outcomes by a non-decreasing value function $u(x)$, and assumes $V($.$) is the mean of the value$ function, namely, $\sum_{i} u\left(x_{i}\right) p_{i}$. The risk attitude of a decision maker under EUT is thus completely determined by the curvature of the value function, where a convex $u($.$) suggests risk-seeking, concave suggests risk aversion, and neither convex nor$ concave suggests risk neutrality. This, however, cannot explain the probabilitydependent risk attitude in our survey. Over the last several decades, there has been abundant evidence showing violations of EUT, and alternative theories, the so-called non-EU models have been proposed.

Some non-EU models use non-linear transformations $w(p)$ of the objective probabilities $p$ in constructing $V($.$) to model people's subjective perceptions of$ probabilities. Some general observations are that people tend to overestimate small probabilities, e.g., the chance of winning a lottery, and underestimate medium to high probabilities, e.g., the number of deaths from relatively frequent causes such as car accidents. For a more detailed description, see the review of non-EU models by Starmer (2000). A commonly accepted inverted S-shaped probability weighting function is due to Tversky and Kahneman (1992):

$$
w(p)=\frac{p^{\delta}}{\left(p^{\delta}+(1-p)^{\delta}\right)^{1 / \delta}}, \delta>0.279 .
$$

Figure 7 illustrates $w(p)$ with $\delta=0.550$ (the red solid line). The blue dotted line shows a perfect perception with $\delta=1$ and $w(p)=p$. A smaller $\delta$ suggests a more 
pronounced inverted S-shape. $w(0)=0$ and $w(1)=1$ suggest that people have no problem perceiving impossibility and certainty. The sensitivity to probability diminishes as we move away from the two extreme points $p=0$ and $p=1$, represented by a flatter curve toward the middle point between 0 and 1 . When $\delta=1$ (the blue dotted line), $w(p)=p$ and we have the conventional EUT model.

\section{Probability Weighting Function}

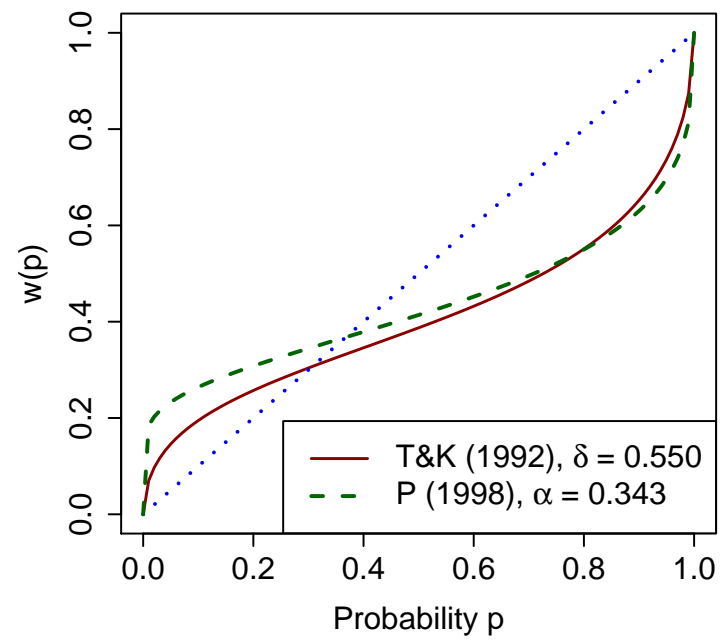

Figure 7: Probability Weighting Functions with Median Estimates

Prelec (1998) proposed another weighting function,

$$
w(p)=\exp \left(-(-\ln p)^{\alpha}\right), 0<\alpha<1,
$$

where a smaller $\alpha$ suggests a more pronounced inverted S-shape, and $\alpha=1$ corresponds to the EUT model with $w(p)=p$. The intersection of the weighting function with the 45-degree line is fixed at $1 / e$ (Figure 7).

The RDEU model further assumes that the decision weight of an outcome also depends on its ranking, e.g., people generally pay relatively more attention to extreme outcomes. The decision weight of outcome $i$ is thus defined as follows:

$$
\pi_{i}=w\left(p_{i}+p_{i+1}+\cdots+p_{m}\right)-w\left(p_{i+1}+\cdots+p_{m}\right), i=1,2, \ldots, m-1
$$

and $\pi_{m}=w\left(p_{m}\right)$.

An additional advantage of the cumulative functional form is that it maintains the monotonicity of $V($.$) , in that V(\mathbf{q})>V\left(\mathbf{q}^{\prime}\right)$ if $\mathbf{q}$ has state-wise stochastic 
dominance over $\mathbf{q}^{\prime}$. Since monotonicity is such an important property that many economists believe should hold in any useful decision theory, RDEU has become one the most popular non-EU theories and is combined with the original prospect theory (Kahneman and Tversky, 1979) to develop the well-known CPT (Tversky and Kahneman, 1992).

A decision maker under the RDEU theory thus maximizes

$$
V(\mathbf{q})=\sum_{i=1}^{m} \pi_{i} u\left(x_{i}\right) .
$$

We further assume a power functional form for $u(x)$ with $x<0$ :

$$
u(x)=-(-x)^{\beta} .
$$

\section{Value Function of Negative Outcomes}

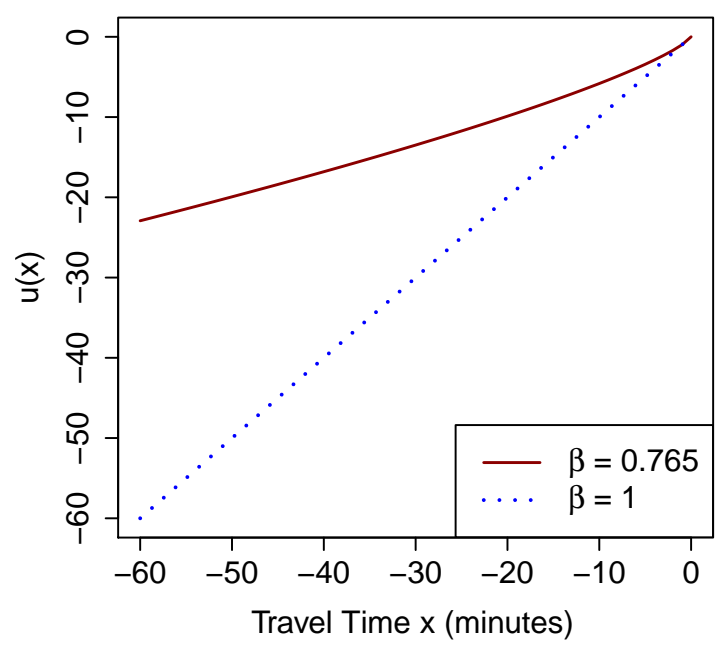

Figure 8: Value Function with $\beta=0.765$

The exponent $\beta$ models how the sensitivity to outcome changes with the magnitude of the outcome. A commonly accepted phenomenon in the economics literature is the diminishing sensitivity $(\beta<1)$. The extension to the perception of travel times is intuitive. For example, the difference between a 10-minute delay and an 11-minute delay would weigh more heavily in a subject's mind than the difference between a 100-minute delay and 101-minute delay, even though the absolute difference for both comparisons is 1 minute. Figure 8 plots such a value 
function with $\beta=0.765$ (the red solid line). As a comparison, $\beta=1$ (the blue dotted line) suggested constant sensitivity to outcome.

\section{Modeling Strategic Behavior}

In this section, we integrate the RDEU model and the random utility model (BenAkiva and Lerman, 1985) so that we can estimate the RDEU parameters along with the probability of strategic route choice from the SP data introduced in Section 2.

Since we are only concerned with the subject's first movement, the relevant choice set consists of two alternatives: the risky branch (Risky) and the safe branch (Safe). Continuing with the assumption laid out in Section 2.1 - that a non-strategic assessment of the risky branch in the strategy map includes the large potential delay, while a strategic assessment ignores it - two separate utility functions can be defined for the risky branch, one for the strategic assessment and one for the non-strategic assessment.

Due to the simplicity of the network, a subject is assumed to exhibit either completely strategic or completely non-strategic behavior in a given scenario. However, over the entire set of scenarios, a given subject may exhibit both strategic and nonstrategic behavior. The labels "strategic" and "non-strategic" therefore, are applied to each choice action, rather than to each individual.

Subjects viewing the network strategically will perceive the possible travel time outcomes of the risky branch as $t_{L}$, the non-delayed travel time of Link $\mathrm{C}$, and $t_{H}$, the fixed travel time of the detour. They know they will never use Link $\mathrm{C}$ in the delayed case. The utility of the risky branch for a strategic (S) individual $n$ in scenario $t$ involving a strategy map with the parameter vector $\phi=\left\{A S C_{R}, \lambda, \beta, \delta\right\}$ is therefore:

$$
V_{n t}(\operatorname{Risky} \mid S, \phi)=A S C_{R}+\lambda\left[\left(t_{H, n t}\right)^{\beta} w\left(p_{n t}\right)+\left(t_{L, n t}\right)^{\beta}\left(1-w\left(p_{n t}\right)\right)\right] .
$$

Note that the negative sign before the power function in Eq. (5) is absorbed by the scale parameter $\lambda$.

Subjects viewing the network non-strategically will perceive the possible outcomes as $t_{L}$ or $t_{M}$, the full delayed travel time of Link C. Since $t_{H}$ is fixed and always higher than $t_{B}$, they would be very unlikely to choose Link D from the outset. Although they may change their paths upon receiving the information, Link D is assumed not to factor into the choice made at the origin. The utility of the risky branch for a non-strategic (NS) individual $n$ in scenario $t$ involving a strategy map is therefore:

$$
V_{n t}(\operatorname{Risky} \mid N S, \boldsymbol{\phi})=A S C_{R}+\lambda\left[\left(t_{M, n t}\right)^{\beta} w\left(p_{n t}\right)+\left(t_{L, n t}\right)^{\beta}\left(1-w\left(p_{n t}\right)\right)\right] .
$$


In both $V_{n t}(\operatorname{Risky} \mid S, \phi)$ and $V_{n t}(\operatorname{Risky} \mid N S, \phi)$, the transformation of the delay probability $w\left(p_{n t}\right)$ follows the probability weighting function in Eq. (1) or (2).

Note that in the simple risk map, there is no difference between strategic and non-strategic utilities, since both see the travel time prospect with $t_{L}$ and $t_{H}$. Thus Eq. (6) applies to both strategic and non-strategic behavior for a scenario in a simple map.

Since there is no risk and no detour on the safe branch (Link B in Figures 1 and 2), the utility of this branch will be identical for strategic and non-strategic subjects:

$$
V_{n t}(\operatorname{Safe} \mid S, \boldsymbol{\phi})=V_{n t}(\operatorname{Safe} \mid N S, \boldsymbol{\phi})=\lambda\left(t_{B, n t}\right)^{\beta} w(1)=\lambda\left(t_{B, n t}\right)^{\beta}
$$

We specify a latent-class mixed Logit model with panel data. The two latent classes, strategic choice and non-strategic choice, are applied at the observation level. The observation-level latent class assumption is supported by the earlier analysis (see Section 2.5) showing that most subjects dsiplayed both strategic and non-strategic behavior during the survey. The value and weighting function parameters of the RDEU model and the risky-branch bias parameter are randomly distributed over individuals.

The utilities are applied within a Logit function to yield the conditional probability of a given choice observation.

$$
P_{n t}\left(i_{t} \mid S, \boldsymbol{\phi}\right)=\frac{\exp \left(V_{n t}\left(i_{t} \mid S, \boldsymbol{\phi}\right)\right)}{\sum_{j_{t}} \exp \left(V_{n t}\left(j_{t} \mid S, \boldsymbol{\phi}\right)\right)}
$$

is the likelihood of individual $n$ choosing alternative $i_{t}$ in scenario $t$, given strategic behavior and parameter vector $\phi$, and

$$
P_{n t}\left(i_{t} \mid N S, \phi\right)=\frac{\exp \left(V_{n t}\left(i_{t} \mid N S, \boldsymbol{\phi}\right)\right)}{\sum_{j_{t}} \exp \left(V_{n t}\left(j_{t} \mid N S, \boldsymbol{\phi}\right)\right)}
$$

is the same likelihood given non-strategic behavior.

The relative proportions of strategic and non-strategic behavior are accounted for with a latent-class model structure, and

$$
P_{n t}\left(i_{t} \mid S, \phi\right) P_{S}+P_{n t}\left(i_{t} \mid N S, \phi\right) P_{N S}
$$

is the likelihood of individual $n$ choosing alternative $i_{t}$ in scenario $t$ given $\phi$ and $P_{S}+P_{N S}=1$. The likelihood of individual $n$ choosing the sequence of observed alternatives over all scenarios, with a given $\phi$ is thus

$$
\prod_{t}\left(P_{n t}\left(i_{t} \mid S, \phi\right) P_{S}+P_{n t}\left(i_{t} \mid N S, \phi\right) P_{N S}\right) .
$$


Variation in taste across subjects is accounted for by assuming a random parameter vector $\phi$ with a normal distribution over subjects. The unconditional likelihood of the sequence of observed alternatives over all scenarios for individual $n$ is the integral of the conditional probability over the distribution of $\phi$ with a probability density function $f(\phi)$ :

$$
\int_{\phi} \prod_{t}\left(P_{n t}\left(i_{t} \mid S, \phi\right) P_{S}+P_{n t}\left(i_{t} \mid N S, \phi\right) P_{N S}\right) f(\phi) d \phi
$$

and the log-likelihood of all observations over all individuals is

$$
\sum_{n} \ln \int_{\phi} \prod_{t}\left(P_{n t}\left(i_{t} \mid S, \phi\right) P_{S}+P_{n t}\left(i_{t} \mid N S, \phi\right) P_{N S}\right) f(\phi) d \phi
$$

which is maximized in the model estimation (Train, 2003).

\section{Results}

All model estimation was performed using BIOGEME Python 2.0 (Bierlaire, 2003; Bierlaire, 2008) with flexible specifications for latent variables. 1000 draws were performed if a simulation was needed for the estimation.

The estimation results are presented in three groups (Tables 1 through 3), representing three types of model specification. Tables 1 and 2 present the conventional linear-in-parameter specifications of systematic utility functions, with summary statistics of random travel times as explanatory variables, specifically travel time mean and standard deviation (STD) in Table 1 and travel time mean and variance (VAR) in Table 2. These represent the commonly used models of travel choice under risk (see, e.g., Lam and Small, 2001; de Palma and Picard, 2006), where travel time variance or standard deviation is used to represent unreliability. Table 3 include models based on the theories of decision under risk from economics, where travel times (and probabilities) are transformed by value (and weighting) functions, and the systematic utility functions are in general not linear in parameters. In all models, two latent classes (strategic and non-strategic) are assumed and the strategic probability is estimated. All random parameters are assumed independent from each other.

\subsection{Mean-Standard-Deviation and Mean-Variance Models}

Three mean-STD models are presented in Table 1. The first is the base model (Mean-STD) with ASC, travel time mean and standard deviation. The second (Mean-STD 1st-order Interaction) and third (Mean-STD 2nd-order Interaction) 


\begin{tabular}{|c||c|c|c|}
\hline & $\begin{array}{c}\text { Mean-STD } \\
\text { (Base 1) }\end{array}$ & $\begin{array}{c}\text { Mean-STD } \\
\text { 1st-order } \\
\text { Interaction }\end{array}$ & $\begin{array}{c}\text { Mean-STD } \\
\text { 2nd-order } \\
\text { Interaction }\end{array}$ \\
\hline \hline Risky Branch & $\mu: 0.0268$ & $\mu:-1.09$ & $\mu:-1.20$ \\
Bias & $(0.133)$ & $(0.175)$ & $(0.171)$ \\
$\boldsymbol{A S C}_{\boldsymbol{R}}$ & $\sigma: 0.558$ & $\sigma: 0.663$ & $\sigma: 0.696$ \\
& $(0.0726)$ & $(0.0778)$ & $(0.0990)$ \\
\hline Mean & $\mu:-0.224$ & $\mu:-0.383$ & $\mu:-0.425$ \\
Travel Time & $(0.0127)$ & $(0.0183)$ & $(0.0217)$ \\
& $\sigma: 0.0380$ & $\sigma: 0.0568$ & $\sigma: 0.0519$ \\
& $(0.0158)$ & $(0.0152)$ & $(0.0180)$ \\
\hline Travel Time & -0.0242 & -0.152 & -0.00960 \\
Std. Dev. (STD) & $(0.00815)$ & $(0.0216)$ & $(0.0256)$ \\
\hline$p \times$ STD & & 0.479 & -0.310 \\
& & $(0.0399)$ & $(0.121)$ \\
\hline$p^{2} \times$ STD & & & $\mu: 0.864$ \\
& & & $(0.132)$ \\
& & & $\sigma: 0.137$ \\
& & & $(0.0290)$ \\
\hline Strategic Prob. & 0.905 & 0.934 & 0.909 \\
$\boldsymbol{P}_{\boldsymbol{S}}$ & $(0.0330)$ & $(0.0250)$ & $(0.0249)$ \\
\hline \hline No. of Ob. & 3466 & 3466 & 3466 \\
\hline No. of Sub. & 74 & 74 & 74 \\
\hline No. of Param. & 6 & 7 & 9 \\
\hline Final LL & -1875.8 & -1656.3 & -1615.2 \\
\hline Adjusted $\rho^{2}$ & 0.217 & 0.308 & 0.324 \\
\hline & & & \\
\hline
\end{tabular}

Table 1: Estimation Results of Mean-STD Models (Values in parentheses are robust standard errors)

models include additional explanatory variables, namely the interactions between the delay probability $(p)$ and the standard deviation (STD), to account for the fact that subjects' risk attitudes vary by the delay probability (Section 2.4). Notice that the interaction models do not have predictive capabilities in general, since it is not clear what delay probabilities should be used when the travel time prospect has more than two outcomes (the extreme case being a prospect with a continuous travel time distribution). In contrast, the RDEU models can be applied to travel time prospects with any number of outcomes, and a continuous extension is also 
available (Connors and Sumalee, 2009). All the random parameters are normally distributed and the distribution parameters $\mu$ (mean) and $\sigma$ (std. dev.) are presented. A fixed parameter indicates that the estimated $\sigma$ is not significantly different from zero in the corresponding unconstrained model.

The parameter to the mean travel time has a negative mean and relatively small standard deviation in each model, suggesting that all subjects view the mean travel time negatively. The Mean-STD model has a slightly negative parameter to the STD, suggesting risk neutrality to slight risk aversion. However, from the preliminary analysis in Section 2.4, we know this is not the complete story. Indeed, the model fit, measured by adjusted $\rho^{2}$, is significantly improved from 0.217 to 0.308 by including an interaction term between the delay probability and STD, which effectively makes the STD parameter $-0.0562,0.0875$, and 0.231 for delay probability of $0.2,0.5$, and 0.8 respectively. Further improvement is realized by including an interaction item between the square of the delay probability and the STD, which effectively makes the average STD parameter $.0370,0.0514$, and 0.295 for delay probability of $0.2,0.5$, and 0.8 respectively. Results from the two interaction models are consistent with the preliminary findings that subjects are risk-averse for low probability, and risk- seeking for high probability. The second-order interaction allows for further fine-tuning of the probability-dependent risk attitude and thus results in a better model fit.

Table 2 presents three mean-VAR models with similar forms to the mean-STD models presented in Table 1. The mean-variance specification is tested due to its potential theoretical appeal, namely that it can be derived from a quadratic value function under the EUT framework (Levy and Markowitz, 1979). There are similar improvements in model fit when the interaction items are included. The two base models, Mean-VAR and Mean-STD, have almost identical model fit, mainly because the effect of STD (and thus VAR) is very close to zero compared to those of the mean travel time and the ASC. When the models are improved with probabilitydependent risk attitudes, the mean-VAR models have much worse fit than the corresponding mean-STD models. This suggests that a mean-STD specification better captures subjects' perception of risks than a mean-VAR one.

\subsection{RDEU Models}

Table 3 first presents the EUT model without probability transformation. This serves as the base model for the four RDEU models, two with the weighting function Eq. (1) from Tversky and Kahneman (1992) and the other two with the weighting function Eq. (2) from Prelec (1998). For the two models with the same weighting function, one has fixed RDEU parameters (exponent of the value function, and the weighting function parameter) while the other has log-normally distributed ran- 


\begin{tabular}{|c||c|c|c|}
\hline & $\begin{array}{c}\text { Mean-VAR } \\
(\text { Base 2) }\end{array}$ & $\begin{array}{c}\text { Mean-VAR } \\
\text { 1st-order } \\
\text { Interaction }\end{array}$ & $\begin{array}{c}\text { Mean-VAR } \\
\text { 2nd-order } \\
\text { Interaction }\end{array}$ \\
\hline \hline Risky Branch & $\mu:-0.147$ & $\mu:-0.988$ & $\mu:-0.872$ \\
Bias & $(0.0856)$ & $(0.110)$ & $(0.116)$ \\
$\boldsymbol{A S C _ { \boldsymbol { R } }}$ & $\sigma: 0.550$ & $\sigma: 0.624$ & $\sigma: 0.622$ \\
& $(0.0158)$ & $(0.0778)$ & $(0.0773)$ \\
\hline Mean & $\mu:-0.224$ & $\mu:-0.307$ & $\mu:-0.340$ \\
Travel Time & $(0.0127)$ & $(0.0128)$ & $(0.0160)$ \\
& $\sigma: 0.0381$ & $\sigma: 0.0462$ & $\sigma: 0.0498$ \\
& $(0.0161)$ & $(0.0142)$ & $(0.0147)$ \\
\hline Travel Time & -0.000603 & -0.00574 & -0.000926 \\
Variance (VAR) & $(0.000202)$ & $(0.000540)$ & $(0.000296)$ \\
\hline$p \times$ VAR & & 0.0251 & -0.0197 \\
& & $(0.00201)$ & $(0.00255)$ \\
\hline$p^{2} \times$ VAR & & & 0.0531 \\
& & & $(0.00425)$ \\
\hline Strategic Prob. & 0.904 & 0.940 & 0.904 \\
$\boldsymbol{P}_{\boldsymbol{S}}$ & $(0.0320)$ & $(0.0252)$ & $(0.0332)$ \\
\hline \hline No. of Ob. & 3466 & 3466 & 3466 \\
\hline No. of Sub. & 74 & 74 & 74 \\
\hline No. of Param. & 6 & 7 & 8 \\
\hline Final LL & -1876.3 & -1748.3 & -1697.4 \\
\hline Adjusted $\rho^{2}$ & 0.217 & 0.269 & 0.290 \\
\hline
\end{tabular}

Table 2: Estimation Results of Mean-VAR models (Values in parentheses are robust standard errors)

dom RDEU parameters that must be non-negative. The distribution parameters $\mu$, mean of $\ln$ (parameter) and $\sigma$, standard deviation of $\ln$ (parameter) are shown for the log-normal distributions.

The value function parameter in the EUT model is greater than one, indicating an increasing sensitivity to travel time outcome, and thus risk aversion. The EUT model however cannot capture the probability-dependent risk attitude, but interestingly the suggested risk aversion is consistent with the two base models from the previous groups (Mean-STD and Mean-VAR) with negative parameters to STD or VAR. The increasing sensitivity to outcome is not consistent with common economic findings (Kahneman and Tversky, 1979), but might be due to the 


\begin{tabular}{|c|c|c|c|c|c|}
\hline & $\begin{array}{c}\text { EUT } \\
\text { (Base 3) }\end{array}$ & $\begin{array}{c}\text { T\&K } \\
\text { (Rand. } \\
\text { Bias) } \\
\end{array}$ & $\begin{array}{c}\text { T\&K } \\
\text { (Rand. Bias\& } \\
\text { RDEU Param.) }\end{array}$ & $\begin{array}{c}\text { Prelec } \\
\text { (Rand. } \\
\text { Bias) } \\
\end{array}$ & $\begin{array}{c}\text { Prelec } \\
\text { (Rand. Bias\& } \\
\text { RDEU Params.) }\end{array}$ \\
\hline $\begin{array}{c}\text { Risky Branch } \\
\text { Bias } \\
A S C_{R}\end{array}$ & $\begin{array}{c}-0.0708 \\
(0.0783)\end{array}$ & $\begin{array}{c}\mu:-1.33 \\
(0.142) \\
\sigma: 0.682 \\
(0.0773)\end{array}$ & $\begin{array}{c}\mu:-1.35 \\
(0.150) \\
\sigma: 0.682 \\
(0.117)\end{array}$ & $\begin{array}{c}\mu:-1.26 \\
(0.130) \\
\sigma: 0.690 \\
(0.0776)\end{array}$ & $\begin{array}{c}\mu:-1.27 \\
(0.131) \\
\sigma: 0.722 \\
(0.0989)\end{array}$ \\
\hline $\begin{array}{c}\text { Scale } \\
\lambda\end{array}$ & $\begin{array}{l}-0.0507 \\
(0.0172)\end{array}$ & $\begin{array}{c}-1.61 \\
(0.622)\end{array}$ & $\begin{array}{c}-1.27 \\
(0.466)\end{array}$ & $\begin{array}{l}-8.55 \\
(4.38)\end{array}$ & $\begin{array}{l}-5.19 \\
(2.86)\end{array}$ \\
\hline $\begin{array}{c}\text { Value Func. } \\
\boldsymbol{\beta}\end{array}$ & $\begin{array}{c}1.31 \\
(0.0756)\end{array}$ & $\begin{array}{c}0.711 \\
(0.0758)\end{array}$ & $\begin{array}{c}\mu:-0.269 \\
(0.0950) \\
\sigma: 0.0515 \\
(0.0153)\end{array}$ & $\begin{array}{c}0.416 \\
(0.0834)\end{array}$ & $\begin{array}{c}\mu:-0.677 \\
(0.191) \\
\sigma: 0.0768 \\
(0.0201)\end{array}$ \\
\hline $\begin{array}{l}\text { Weight. Func. } \\
\text { (T\&K) } \\
\delta\end{array}$ & & $\begin{array}{c}0.545 \\
(0.0174)\end{array}$ & $\begin{array}{l}\mu:-0.597 \\
(0.0405) \\
\sigma: 0.144 \\
(0.0410)\end{array}$ & & \\
\hline $\begin{array}{c}\text { Weight. Func. } \\
\text { (Prelec) } \\
\alpha\end{array}$ & & & & $\begin{array}{c}0.367 \\
(0.0277)\end{array}$ & $\begin{array}{c}\mu:-1.07 \\
(0.0785) \\
\sigma:-0.491 \\
(0.0776)\end{array}$ \\
\hline $\begin{array}{c}\text { Strategic Prob. } \\
\boldsymbol{P}_{S}\end{array}$ & $\begin{array}{c}0.880 \\
(0.0333)\end{array}$ & $\begin{array}{c}0.921 \\
(0.0251)\end{array}$ & $\begin{array}{c}0.925 \\
(0.0231)\end{array}$ & $\begin{array}{c}0.927 \\
(0.0251)\end{array}$ & $\begin{array}{c}0.934 \\
(0.0231)\end{array}$ \\
\hline No. of Ob. & 3466 & 3466 & 3466 & 3466 & 3466 \\
\hline No. of Sub. & & 74 & 74 & 74 & 74 \\
\hline No. of Param. & 4 & 6 & 8 & 6 & 8 \\
\hline Final LL & -1916.1 & -1644.8 & -1634.4 & -1632.2 & -1608.4 \\
\hline Adjusted $\rho^{2}$ & 0.200 & 0.313 & 0.316 & 0.318 & 0.327 \\
\hline
\end{tabular}

Table 3: Estimation Results of RDEU Models (Values in parentheses are robust standard errors)

mis-specification of the model.

Significant model fit improvements are observed in the RDEU models compared to the EUT model, suggesting significant distortions of outcome probabilities. Estimation results for the scale parameter $\lambda$ show a significantly negative effect of the RDEU model component (one-sided at the 5\% level). The improvement in model fit achieved through random RDEU parameters ( 0.313 to 0.316 with 
T\&K, and 0.318 to 0.327 with Prelec) suggests a certain level of taste variations over individuals in valuing travel time outcomes and weighting probabilities.

\begin{tabular}{|c|cccc|}
\hline & Mean & Median & Mode & Std. Dev. \\
\hline Value Func. $\beta$ & 0.765 & 0.764 & 0.762 & 0.040 \\
\hline Weighting Func. $\delta$ & 0.556 & 0.550 & 0.539 & 0.081 \\
\hline
\end{tabular}

Table 4: Statistics for Log-normally distributed RDEU parameters (Eq. 1, Tversky \& Kahneman, 1992)

\begin{tabular}{|c|cccc|}
\hline & Mean & Median & Mode & Std. Dev. \\
\hline Value Func. $\beta$ & 0.510 & 0.508 & 0.505 & 0.039 \\
\hline Weighting Func. $\alpha$ & 0.387 & 0.343 & 0.279 & 0.202 \\
\hline
\end{tabular}

Table 5: Statistics for Log-normally distributed RDEU parameters (Eq. 2, Prelec, 1998)

Tables 4 and 5 show the relevant statistics of the log-normally distributed RDEU parameters. The estimates for the value parameter $\beta$ indicate a significant decrease in sensitivity to travel times. The relatively small standard deviation $(0.040$ and 0.039 for the T\&K and Prelec models respectively) of the parameter over all subjects suggests that very few if any subjects displayed increasing sensitivity to travel times, as would be the case for $\beta>1$.

Estimates for the probability weighting parameters $\delta$ and $\alpha$ are significantly smaller than one for virtually all subjects, and are well in line with expectations, suggesting substantial overweighting of high probabilities and underweighting of low probabilities. Under RDEU, as the probabilities enter the preference function non-linearly, the risk attitude is determined jointly by the value function $u($.$) and$ probability weighting function $w($.$) . A convex u().(\beta<1)$ would have suggested risk-seeking regardless of the outcome probabilities if EUT is applied. However, under RDEU, the decision weight of the more extreme travel time (the delayed travel time on the risky branch) is $w(p)$ and that of the regular travel time $1-w(p)$. On the one hand, when the delay probability $p$ is small $(0.2), w(0.2)>0.2$ and $1-w(0.2)<0.8$, suggesting that the travel delay weighs more than proportionally compared with the regular travel time. This reflects pessimism in perceiving the chance of delay and if there is enough pessimism ( $\delta$ is small enough), a subject might exhibit risk aversion even with a convex $u($.$) , as is the case in our survey.$ On the other hand, when the delay probability is large $(0.8), w(0.8)<0.8$ and 
$1-w(0.8)>0.2$, suggesting that the travel delay weighs less than proportionally compared with the regular travel time. This reflects optimism in perceiving the chance of delay and strengthens risk-seeking on the basis of a convex $u($.$) . To sum$ up, the estimated RDEU parameters support our risk attitude findings reported in Section 2.4 .
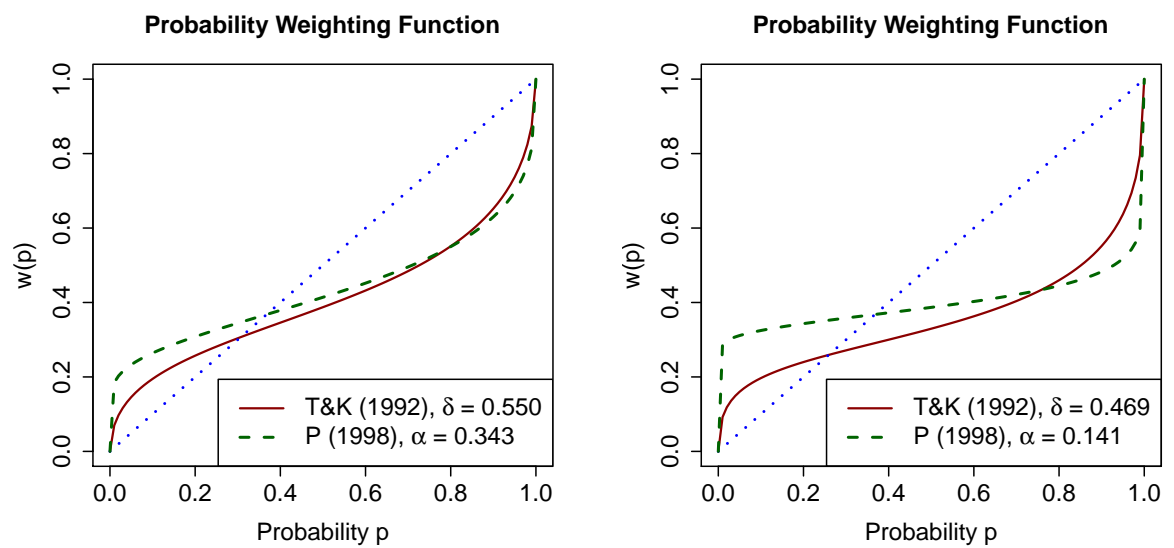

(a) With Median Estimates (a repetition of Fig- (b) With Estimates One STD below the Median ure 7)

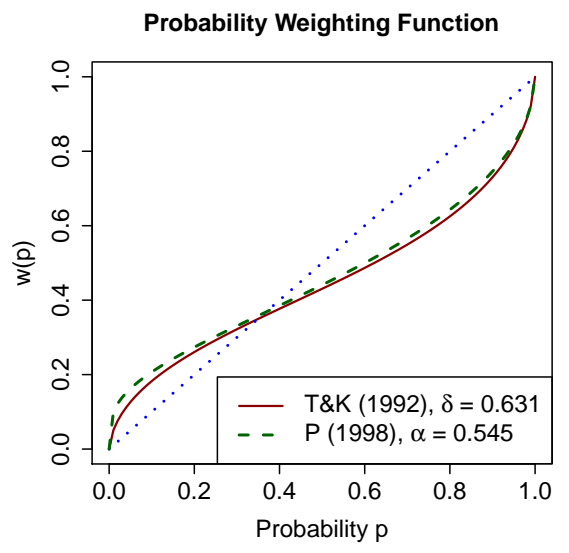

(c) With Estimates One STD above the Median

Figure 9: Probability Weighting Functions with Three Different Sets of Parameters

We take a further look at the T\&K and Prelec weighting functions. Figure 9(a) (a repetition of Figure 7) shows the two weighting functions with the median estimates of $\delta$ and $\alpha$. Figures 9(b) and 9(c) show the weighting functions with $\delta$ 
and $\alpha$ at one standard deviation below and above the median estimate respectively. We see relatively small difference between the two weighting functions when the parameters are at the median or one standard deviation above the media, but significant difference with a delay probability of 0.2 or 0.5 when the parameters are one standard deviation below the median. Specifically the Prelec function gives higher decision weights to 0.2 or 0.5 , and accordingly a more significant risk aversion at the two lower probability levels.

All RDEU models estimate a large and significant bias against the risky branch. The parameter $A S C_{R}$ accounts for preference factors not directly related to travel time or risk. The large estimated standard deviation suggests a wide variation in subjects' inherent preference, but the vast majority of subjects appear to have a preference for the safe branch. One possible explanation for this preference is that the safe branch is straightforward, requiring no interpretation of probability or outcomes. Another possible explanation is that, in the interface presented, the risky branch required two mouse clicks to traverse (one to reach the information sign and one to complete the trip). The safe branch required only one click. This difference may have deterred some users from choosing the risky branch unless the potential benefits were especially clear.

The estimated probability of strategic choice is robust across all models and significantly different from both 0 and 1 , suggesting that a latent-class model is indeed necessary. This is also somewhat consistent with the preliminary analysis in Section 2.5, where $15 \%$ of choice pairs are distinguishably non-strategic.

Finally we compare the RDEU model specifications with the conventional linear-in-parameter specifications. As the purpose of model development is to predict travel choices, the interaction models from the Mean-STD and Mean-VAR groups are not generally applicable due to the lack of predictive capabilities. In this sense, the RDEU models are far superior with a best model fit of 0.327 compared to 0.217 of the Mean-STD model. Even if we ignore the predictive capability, the best Mean-STD model fit (0.324) is still not as good. Furthermore, the RDEU model parameters have clear interpretations, representing sensitivities to outcomes and probabilities. The Mean-STD interaction models, however, seem to simply fit the data without providing much insight into the underlying behavior.

\section{Conclusions and Future Directions}

This paper incorporates the RDEU theory under the random utility modeling framework for strategic route choice under real-time traffic information using SP data. The use of RDEU is motivated by the preliminary analysis of the SP data showing that a traveler's risk attitude depends on the probability of an outcome in a risky 
travel time prospect.

We specify a latent-class mixed Logit model for panel data with two latent classes, strategic and non-strategic route choice. The class probability is applied at the observation level, since preliminary analysis of the data shows that the same individual can be strategic in one situation and non-strategic in another. The strategic class probability is significantly different from both 0 and 1, which suggests that both strategic and non-strategic route choice behavior exist and a proper route choice model under real-time traffic information should consider both. Two RDEU parameters are treated as lognormal random variables distributed over individuals. The exponent of the value function is less than one for almost all travelers, suggesting a diminishing sensitivity to travel time, which is both intuitive and consistent with the economics literature where outcomes are measured by monetary payoffs. The probability weighting function parameter is less than one for almost all travelers, suggesting a pronounced inverted S-shaped curve. Together, the value and weighting functions, with their estimated parameter values, explain the observation of probability-dependent risk attitude.

The current SP survey contains one-shot decision making tasks where the probabilities of the outcomes are described to the subjects. In reality, however, likely learn the chances of probabilistic outcomes over time, through a combination of their own experience, traffic reports, and word-of-mouth. Barron and Erev (2003) first demonstrates the difference between experience-based and description-based decision making and later Ben-Elia et al. (2008) confirms the difference in a travel choice context. Rakow and Newell (2010) provides a good overview of this topic. In future research we will collect more realistic route choice data where explicit learning is allowed in an SP, field experiment, and/or revealed preference (RP) context and develop the corresponding modeling methodologies.

$\mathrm{CPT}$ evaluates outcomes with respect to a reference point and treats outcomes as losses or gains, in addition to being rank-dependent. Whether CPT is a better model for the current SP data is an interesting future research topic. The key challenge is the determination of the reference point, which likely varies across individuals and possibly across choice situations. A possible solution is to specify a number of latent classes, each for a different reference point, and estimate each class probability. This adds to the complexity of the current model, which already has one latent variable to measure strategic probability. Greater data variability is required to estimate latent variables and if the current data set does not meet the requirement, additional data can be collected. A fuzzy reference point approach is proposed by Avineri (2009) and worth further exploration.

Model forms beyond RDEU and CPT must also be tested in the context of strategic route choice. One relatively unexplored avenue is the application of process models, such as the Priority Heuristic approach proposed by Brandstatter et al. 
(2006). Process models attempt to model the actual decision-making process of an individual traveler. This differs from "as-if" modeling frameworks, such as RDEU and CPT, which simply seek to predict the likelihood of a choice, without attempting to replicate the decision process.

The strategic route choice model will also be applied to a real-life network with a general structure. A latent-choice model that is applicable in a general network is specified in Gao et al. (2008) to take into account the fact that a strategy is a plan in the traveler's mind and only the realized path is observed. The major obstacle to real-world application is the lack of data on travelers' real-time traffic information access, as the choice set of strategic alternatives is closely related to what type of information a traveler has. The issue could be resolved by more advanced data collection technologies, and/or more advanced modeling techniques such as latent classes or even continuous distributions of real-time information access.

\section{References}

Abdel-Aty, M. A. and Abdalla, M. F. (2006). Examination of multiple mode/routechoice paradigms under atis, IEEE Transactions on Intelligent Transportation Systems 7(3): 332-348.

Avineri, E. (2004). A cumulative prospect theory approach to passengers behavior modeling: Waiting time paradox revisited, Journal of Intelligent Transportation Systems 8: 195-204.

Avineri, E. (2009). Incorporating fuzzy reference points into applications of travel choice modeling, in E. Avineri, M. Koppen, K. Dahal, Y. Sunitiyoso and R. Roy (eds), Applications of Soft Computing, Vol. 52 of Advances in Intelligent and Soft Computing, Springer-Verlag, Heidelberg, pp. 221-229.

Avineri, E. and Prashker, J. N. (2004). Violations of expected utility theory in route-choice stated preferences, Transportation Research Record: Journal of the Transportation Research Board 1894: 222-229.

Avineri, E. and Prashker, J. N. (2005). Sensitivity to travel time variability: Travelers' learningn perspective, Transportation Research Part C 13: 157-183.

Barron, G. and Erev, I. (2003). Small feedback-based decisions and their limited correspondence to description-based decisions, Journal of Behavioral Decision Making 16: 215-233.

Ben-Akiva, M. and Lerman, S. (1985). Discrete Choice Analysis, MIT Press. 
Ben-Elia, E., Erev, I. and Shiftan, Y. (2008). The combined effect of information and experience on drivers' route-choice behavior, Transportation 35: 165177.

Ben-Elia, E. and Shiftan, Y. (2010). Which road do i take? a learning-based model of route-choice behavior with real-time information, Transportation Research Part A 44: 249-264.

Bierlaire, M. (2003). Biogeme: a free package for the estimation of discrete choice models, Proceedings of the 3rd Swiss Transport Research Conference, Ascona, Switzerland.

Bierlaire, M. (2008). An introduction to BIOGEME version 1.6. http://biogeme.epfl.ch.

Brandstatter, E., Gigerenzer, G. and Hertwig, R. (2006). The priority heuristic: Making choices without trade-offs, Psychological Review 113(2): 409-432.

Chorus, C. G., Molin, E. J. E. and Van Wee, B. (2006). Use and effects of advanced traveller information services (atis): A review of the literature, Transport Review 26(2): 127-149.

Connors, R. D. and Sumalee, A. (2009). A network equilibrium model with travellers' perception of stochastic travel times, Transportation Research Part B 43: 614-624.

de Lapparent, M. (2010). Attitude toward risk of time loss in travel activity and air route choices, Journal of Intelligent Transportation Systems 14(3): 166-178.

de Palma, A., Ben-Akiva, M., Brownstone, D., Holt, C., Magnac, T., McFadden, D., Moffatt, P., Picard, N., Train, K., Wakker, P. and Walker, J. (2008). Risk, uncertainty and discrete choice models, Marketing Letters 19: 269-285.

de Palma, A. and Picard, N. (2006). Equilibria and information provision in risky networks with risk-averse drivers, Transportation Science 40(4): 393-408.

Gao, S., Frejinger, E. and Ben-Akiva, M. (2008). Adaptive route choice models in stochastic time-dependent networks, Transportation Research Record: Journal of the Transportation Research Board 2085: 136-143.

Gao, S., Frejinger, E. and Ben-Akiva, M. (2010). Adaptive route choices in risky traffic networks: A prospect theory approach, Transportation Research Part C 18: 727-740. 
Jou, R.-C., Kitamura, R., Weng, M.-C. and Chen, C.-C. (2008). Dynamic commuter departure time choice under uncertainty, Transportation Research Part A 42: 774-783.

Kahneman, D. and Tversky, A. (1979). Prospect theory: An analysis of decision under risk, Econometrica 47(2): 263-292.

Katsikopoulos, K. V., Duse-Anthony, Y., Fisher, D. L. and Duffy, S. A. (2000). The framing of drivers' route choices when travel time information is provided under varying degrees of cognitive load, Human Factors 42(3): 470-481.

Katsikopoulos, K. V., Duse-Anthony, Y., Fisher, D. L. and Duffy, S. A. (2002). Risk attitude reverslas in drivers' route choice when range of travel time information is provided, Human Factors 44(3): 466-473.

Lam, T. C. and Small, K. A. (2001). The value of time and reliability: measurement from a value pricing experiment, Transportation Research Part E 37: 231251.

Levy, H. and Markowitz, H. M. (1979). Approximating expected utility by a function of mean and variance, American Economic Review 69(3): 308-317.

Prelec, D. (1998). The probability weighting function, Econometrica 66(3): 497527.

Quiggin, J. (1982). A theory of anticipated utility, Journal of Economic Behavior and Organization 3(4): 497-527.

Rakow, T. and Newell, B. R. (2010). Degree of uncertainty: An overview and framework for future resesarch on experience-based choice, Journal of Behavioral Decision Making 23: 1-14.

Razo, M. and Gao, S. (2010). Strategic thinking and risk attitudes in route choice: Stated preference approach, Transportation Research Record: Journal of the Transportation Research Board 2156: 28-35.

Schmeidler, D. (1989). Subjective probability and expected utility without additivity, Econometrica 57: 571-587.

Schwanen, T. and Ettema, D. (2009). Coping with unreliable transportation when collecting children: examiningn parents' behavior with cumulative prospect theory, Transportation Research Part C pp. 511-525. 
Senbil, M. and Kitamura, R. (2004). Reference points in commuter departure time choice: A prospect theoretic test of alternative decision frames, Journal of Intelligent Transportation Systems 8: 19-31.

Starmer, C. (2000). Developments in non-expected utility theory: The hunt for a descriptive theory of choice under risk, Journal of Economic Literature XXXVIII: $332-382$.

Train, K. (2003). Discrete Choice Methods with Simulation, Cambridge University Press.

Tversky, A. and Kahneman, D. (1992). Advances in prospect theory: Cumulative representation of uncertainty, Journal of Risk and Uncertainty 5: 297-323.

$\mathrm{Xu}, \mathrm{H}$. , Zhou, J. and Xu, W. (2010). A decision-making rule for modeling travelers' route choice behavior based on cumulative prospect theory, Transportation Research Part C doi:10.1016/j.trc.2010.05.009. 